\title{
No evidence to implicate Borrelia burgdorferi in the pathogenesis of dilated cardiomyopathy in the United Kingdom
}

\author{
D H E Rees, P J Keeling, W J McKenna, J S Axford
}

\begin{abstract}
Objective-To determine whether Borrelia burgdorferi is implicated in the pathogenesis of dilated cardiomyopathy in the United Kingdom.

Design-A controlled prospective study. Patients' notes were reviewed for evidence of Lyme disease and serum samples were tested by enzyme linked immunoadsorbent assay (ELISA) for antibodies to $B$ burgdorferi. Samples with raised antibody concentrations were subsequently analysed by immunoblotting to determine their antibody binding specificity.

Setting-Tertiary referral centre.
\end{abstract}

Patients-97 consecutive patients with dilated cardiomyopathy diagnosed according to World Health Organisation criteria were studied. Serum samples were taken from two matched control groups. The first group ( $n=38$ ) was age, sex, and geographically matched. The second control group ( $=39$ ) was environmentally matched and consisted of members of the patients' own households.

Main outcome measures-Clinical evidence of Lyme disease. Presence of raised antibody concentrations to $B$ burgdorferi.

Results-No patients had a previous illness compatible with Lyme disease. Analysis of the ELISA data showed eight of 97 patients with dilated cardiomyopathy $(8 \cdot 2 \%)$ and two of 77 controls (3.9\%) had raised antibody concentrations. Immunoblot analysis, however, did not show binding patterns consistent with the presence of IgG specific for $B$ burgdorferi in any of these samples.

Conclusions-There was no clinical or serological evidence to implicate $B$ burgdorferi in the pathogenesis of idiopathic dilated cardiomyopathy in the United Kingdom. In the absence of specific symptoms or likely exposure to $B$ burgdorferi routine serological testing for Lyme disease in this group of patients is not recommended. Furthermore, raised antibodies to $B$ burgdorferi are not diagnostic of active infection and ELISA results should be interpreted with caution unless specific $B$ burgdorferi antibody bands have been found by immunoblot analysis.

(Br Heart F 1994;71:459-461)
Lyme disease is endemic in several regions of the United Kingdom. ${ }^{1}$ The initial manifestations of the disease are the characteristic skin lesion, erythema migrans, and symptoms of general malaise (stage 1). This may be followed weeks to months later by acute articular, cardiac, and neurological manifestations (stage II). Months to years later intermittent or continuous attacks of mono/oligoarthritis may occur (stage III). Nearly half of all patients with stage II and III Lyme disease, however, do not report a preceding skin rash or tick bite. ${ }^{2}$

Lyme carditis was first described in the United States in $1980^{3}$ and in Europe in 1984. ${ }^{4}$ Histological changes have been described $^{5}$ and spirochaetes have been isolated from endomyocardial biopsy specimens. ${ }^{6}$ Cardiac involvement has mainly been reported as myocarditis, pericarditis, and conduction disorders, occurring in $1 \%-8 \%$ of cases of Lyme disease. ${ }^{6}$ It usually occurs three to six weeks after the initial illness, although cardiac manifestations may be the sole presenting feature of the condition, ${ }^{7}$ occurring without a history of erythema migrans or tick bite. In a recent review of 105 cases of Lyme carditis in the United States and Europe ${ }^{8}$ cardiac involvement mainly consisted of acute myocarditis and conduction disease with nearly all patients making a full recovery and only one patient developing permanent atrioventricular block. Of note, only one third remembered a preceeding tick bite and two thirds reported the characteristic rash of erythema migrans, although this was less common in Europe $(58 \%)$ than in the United States $(82 \%)$

Recently Lyme disease has been implicated in the pathogenesis of some cases of dilated cardiomyopathy. In 1991 Borrelia burgdorferi was isolated, for the first time, from a patient with established dilated cardiomyopathy. ${ }^{9}$ A subsequent report, with an enzyme linked immunosorbent assay (ELISA) to detect antibodies to $B$ burgdorferi, showed a significantly higher prevalence of raised antibodies in patients with dilated cardiomyopathy than in control groups. ${ }^{10}$ Since then, two further studies have reported evidence of infection with $B$ burgdorferi in patients with dilated cardiomyopathy. The first found evidence of active infection with $B$ burgdorferi in patients with established dilated cardiomyopathy by silver staining or culture of the spirochaetes from endomyocardial biopsies, but found a poor response to antibiotic treatment. ${ }^{11}$ The second group only found serological evidence 
of infection and reported improvement after antibiotic treatment in eight out of nine of these patients ${ }^{12}$; however they failed to mention the duration of dilated cardiomyopathy in their study and the findings should be interpreted with caution as raised antibody titres are not diagnostic of active infection. ${ }^{13}$

As $B$ burgdorferi has been implicated in the pathogenesis of dilated cardiomyopathy and cardiac manifestations may be the sole presentation of Lyme disease we considered it important to determine whether there was any clinical or serological evidence for infection with $B$ burgdorferi in patients from the United Kingdom with idiopathic dilated cardiomyopathy.

\section{Patients and methods}

\section{PATIENTS WITH DILATED CARDIOMYOPATHY}

The study group consisted of 97 consecutive patients with dilated cardiomyopathy (mean (range) age 43 (12-74), 71 male) referred to our centre from several locations: Greater London ( $n=38)$, south east counties ( $n=$ 31), Hampshire $(n=5)$, Wales $(n=7)$, west country $(n=5)$, Italy and Greece $(n=5)$, Yorkshire $(n=4)$, and Jersey $(n=2)$. None of the patients were at occupational risk of exposure to tick bites-for example, farmers, forrestry workers, and park workers. The clinical diagnosis of dilated cardiomyopathy was made according to World Health Organisation criteria, ${ }^{14}$ and all patients underwent cardiac catheterisation and right ventricular endomyocardial biopsy. Patients had been symptomatic for a mean (SD) duration of $34(48)$ months, $30(31 \%)$ described an acute viral illness at disease onset, and 13 $(14 \%)$ had either clinical $(n=7)$ or histological $(n=6)$ evidence of myocarditis. ${ }^{15}$

\section{CONTROLS}

Serum samples were obtained from two matched control groups. The first control group ( $n=38$; mean (range) age $45(14-72$ ), 34 male) was age, sex, and geographically matched by selecting the next person on the patient's own general practitioner's list with the same sex and within five years of the patient's age. Subjects of the second control group ( $n=39$; mean (range) age $42(7-68)$, 12 male) were environmentally matched and each consisted of a member of the patient's own household, who was usually the spouse $(n=32)$, but occasionally a parent $(n=5)$, or child $(n=2)$.

\section{NORMAL POPULATION}

Serum samples from healthy blood donors ( $n$ $=50$; mean (range) age 45, (20-70), 25 male) was used to determine the normal range for IgG $B$ burgdorferi antibodies in a non-susceptible population.

\section{Methods}

The patients' notes were reviewed for clinical evidence of Lyme disease. Antibodies to $B$ burgdorferi were detected by ELISA with sonicated whole cell spirochaetes (strain B31) as antigen, and samples with raised antibody concentrations were analysed by immunoblot to further define antibody specificity.

\section{Enzyme linked immunosorbent assay}

Half of 96-well Immulon 1 immunoassay plates (Dynatech) were coated with $50 \mu 1$ of a 5 $\mu \mathrm{g} / \mathrm{ml}$ suspension of sonicated spirochaetes in carbonate buffer ( $\mathrm{pH} 9.6)$ by incubation overnight at $4^{\circ} \mathrm{C}$. The plates were subsequently blocked with $3 \%$ bovine serum albumin in phosphate buffered saline for one hour at $37^{\circ} \mathrm{C}$. Serum samples were diluted one in 800 in phosphate buffered saline containing $0.05 \%$ Tween-80 (BDH), 3\% goat serum, and $1 \%$ bovine serum albumin. Each sample $(100 \mu \mathrm{l})$ was added in triplicate to both the antigen coated wells and uncoated control wells and incubated for one hour at $37^{\circ} \mathrm{C}$. The same sample from a patient with raised antibody concentrations (positive control) was also added in triplicate to each plate. After washing, the wells were further incubated for one hour at $37^{\circ} \mathrm{C}$ with goat antihuman IgG-alkaline phosphatase conjugate (Sigma Chemicals). Antibody binding was detected with $p$-nitrophenyl phosphate substrate (Sigma Chemicals) and the optical density was read at $405 \mathrm{~nm}$. Background binding to the uncoated control wells was subtracted from the readings and results were expressed as a ratio of the mean optical density of the test sample to the positive control.

\section{Immunoblott analysis}

Borrelia burgdorferi proteins (strain B31) were separated on a $10 \%$ polyacrylamide gel as described elsewhere, ${ }^{16}$ and were transferred on to a nitrocellulose membrane by semi-dry blotting. Excess binding sites were blocked by incubation in 5\% dried skimmed milk in phosphate buffered saline. Strips cut from the nitrocellulose were incubated for one hour at $37^{\circ} \mathrm{C}$ in serum diluted one in 500 in phosphate buffered saline containing $5 \%$ dried skimmed milk and $0 \cdot 2 \%$ Tween-80. After washing, the strips were incubated for one hour at $37^{\circ} \mathrm{C}$ with biotinylated goat antihuman IgG (Amersham) and for a further hour in streptavidin horseradish peroxidase (Amersham), both were diluted one in 1000 with phosphate buffered saline-Tween, and antibody binding was subsequently detected by the enhanced chemiluminescence system (Amersham). Binding of antibody to any of 14 protein bands with molecular weights of $17,22,29,31,34,39,41,46,55,60,66,75$, 83,94 kilodaltons $(\mathrm{kDa})$ previously reported to be associated with $B$ burgdorferi, ${ }^{17}$ was assessed blindly by an experienced and independent observer.

Detection of antibodies to Treponema pallidum Samples with raised $B$ burgdorferi antibody concentrations by ELISA $(n=11)$ were screened for binding to $T$ pallidum by the $T$ pallidum haemagglutination (TPHA) test and the venereal disease reference laboratory (VDRL) test.

\section{STATISTICAL ANALYSIS}

A serum sample was defined as positive for antibodies to $B$ burgdorferi by ELISA if the 
optical density ratio was greater than the mean optical density ratio +2 SDs of the normal population. A $\chi^{2}$ probability test with Yate's correction was used to compare differences in the number of positive samples in the dilated cardiomyopathy and control groups.

\section{Results}

CLINICAL

On reviewing the notes, none of the patients had either a documented rash consistent with erythema migrans or a previous illness compatible with Lyme disease.

\section{DETECTION OF ANTIBODIES}

Analysis of the ELISA data showed the presence of raised antibodies to $B$ burgdorferi in eight of $97(8.2 \%)$ patients with dilated cardiomyopathy, two of $38(5 \cdot 3 \%)$ geographically matched controls, and one of $39(2 \cdot 6 \%)$ environmentally matched controls. There was no significant difference between the dilated cadiomyopathy group and either the geographically matched controls $\left(\chi^{2}=0.03\right.$, $\mathrm{p}=0 \cdot 8$ ), environmentally matched controls $\left(\chi^{2}=0.6, \mathrm{p}=0.4\right)$, or the control group overall $\left(\chi^{2}=0.736 ; p=0.4\right)$. Immunoblot analysis showed only very weak antibody binding to the $60 \mathrm{kDa}$ protein in one patient with dilated cardiomyopathy, to the $17 \mathrm{kDa}$ protein in a further patient and 17 and $20 \mathrm{kDa}$ in one other. One of the controls had very weak binding to 30 and $41 \mathrm{kDa}$ proteins and another to the $41 \mathrm{kDa}$ protein. These patterns of antibody binding with weak reactivity do not indicate active infection with $B$ burgdorferi and most likely result from the presence of cross reacting antibodies.

\section{Antibodies to Treponema pallidum}

One sample was positive in the TPHA test and no samples tested were positive in the VDRL test.

\section{Discussion}

It is likely from the evidence reviewed that $B$ burgdorferi is the cause of some cases of dilated cardiomyopathy in western Europe. In this study we have investigated, for the first time in the United Kingdom, patients with idiopathic dilated cardiomyopathy for clinical and serological evidence of infection with $B$ burgdorferi. The group studied were referred to a tertiary referral centre in southern England, including 17 patients (18\%) from regions (Hampshire, Wales, Norfolk, and the west country) in which ticks infected with $B$ burgdorferi have been identified ${ }^{18}$ or cases of Lyme disease reported. ${ }^{1}$ Furthermore, patients not resident in these areas, may have visited endemic areas for Lyme disease within this country, Europe, or North America. Of note, none of the patients with dilated cardiomyopathy were at occupational risk of exposure to tick bites.

In this study group, we found no clinical evidence of Lyme disease and although $8.2 \%$ of the patients with dilated cardiomyopathy tested had raised serum $B$ burgdorferi antibody concentrations on ELISA, this was not signifi- cantly more than in the control groups and subsequent immunoblot analysis did not show antibody binding patterns consistent with $B$ burgdorferi infection in any of the samples. This lack of specificity with ELISA is consistent with other published data. ${ }^{13} 19$ False positive antibody concentrations to $T$ pallidum, a potentially cross reactive infection ${ }^{20}$ only occurred in one patient.

In conclusion, from this group of patients (some of whom live in or near endemic areas for Lyme disease), we have found no evidence to implicate $B$ burgdorferi in the pathogenesis of idiopathic dilated cardiomyopathy in the United Kingdom. In the absence of specific symptoms or likely exposure to $B$ burgdorferi we would not recommend routine serological testing for Lyme disease in these patients. Our data also confirm that ELISA results should be interpreted with caution unless specific $B$ burgdorferi antibody bands have been found on immunoblot analysis. Furthermore, raised antibodies to $B$ burgdorferi are not necessarily indicative of active cardiac infection, which can only be diagnosed with certainty by the presence of the spirochaetes in endomyocardial biopsies by culture or detection of $B$ burgdorferi DNA by the polymerase chain reaction.

1 Muhleman MF, Wright DJM. Emerging pattern of Lyme disease in the United Kingdom and Irish Republic. Lancet 1987;1:260-2.

2 Bianchi G, Rovetta G, Monteforte P, Fumarola D, Trevisan G, Crovato F, et al. Articular involvement in European Lyme disease. A report of 32 Italian patients. Br $\mathcal{F}$ Rheumatol 1990;29:178-80.

3 Steere AC, Batsford WP, Weinberg M, et al. Lyme carditis: cardiac abnormalities of Lyme disease. Ann Intern Med 1980;93:8-16.

4 Houwerzijl J, Root J, Hoogkamp-Korstanje JAA. A case of Lyme disease with cardiac involvement in the Lyme disease with cardiac in

5 Reznick JW, Braunstein DB, Walsh RL, et al. Lyme carditis. Electrophysiologic and histopathologic study. Am $\mathcal{f}$ Med 1986;81:923-7.

6 De Koning J, Hoogkamp-Korstanje JAA, Van der Linde MR, Crijns M, et al. Demonstration of spirochaetes in cardiac biopsies of patients with Lyme disease. F Infect Dis 1989;160:150-3.

7 Vlay SC. Complete heart block due to Lyme disease. $N$ Engl $\mathcal{F}$ Med 1986;315:1418.

8 Van der Linde MR. Lyme carditis: clinical characteristics of 105 cases. Scand f Infect Dis 1991;77:81-4.

9 Stanek G, Klein J, Bittner R, Glogar D. Isolation of Borrelia burgdorferi from the myocardium of a patien with longstanding cardiomyopathy. $N$ Engl $\mathscr{f} \mathrm{Med}$ 1990;322:249-52.

10 Stanek G, Klein J, Bittner R, Glogar D. Borrelia burgdorferi as an etiologic agent in chronic heart failure. Scand $\mathcal{F}$ Infect Dis 1991;77:85-7.

11 Bergler-Klein J, Glogar D, Stanek G. Clinical outcome of Borrelia burgdorferi related dilated cardiomyopathy after Borrelia burgdorfen related dilated cardiomyopathy af
antibiotic treatment (letter). Lancet 1992;340:317-8.

12 Gasser R, Dusleag J, Reisinger E, et al. Reversal by ceftriaxone of dilated cardiomyopathy Borrelia burgdorferi axone of dilated cardiomyopathy Borrelia

13 Haywood GA, O'Connell S, Gray HH. Lyme carditis: a United Kingdom perspective. Br Heart f 1993;70:15-6.

14 Brandenburg RO, Chazov E, Cherian G, et al. Report of the WHO/ISPC task force on definition and classification of the cardiomyopathies. Circulation 1981;64 1397-9.

15 Anetz HT, Billingham ME, Edwards WD, et al. Myocarditis, a histopathologic definition and classification. Am f Cardiovasc Pathol 1987;1:3-14.

16 Laemmli UK. Cleavage of structural proteins during the assembly of the head of bacteriophage T4. Nature 1974;227:680-5.

17 Bingnan MA, Christen B, Leung D, Vigo-Pelfrey C. Serodiagnosis of Lyme Borreliosis by western immunoblot: reactivity of various significant antibodie against

18 Nuttall P, Randolph S, Carey D, Craine N, Livesley A. Ecology of Lyme Borreliosis in the United Kingdom. Ann Rheum Dis 1993;52:394-6.

19 Berg D, Abson KG, Prose NS. The laboratory diagnosis of Lyme disease. Arch dermatol 1991;127:866-70.

20 Magnarelli LA, Miller JN, Anderson JF, Riviere GR Cross-reactivity of non specific treponemal antibody in Cross-reactivity of non specific treponemal antibody in
serological tests for Lyme disease. $\mathcal{f}$ Clin Microbiol 1990; 28:1276-9. 